\title{
Comprehensive Analysis of State-of- the-Art CAD Tools and Techniques for Chronic Kidney Disease (CKD)
}

\author{
Mynapati Lakshmi Prasudha, VNR Vignana Jyothi Institute of Engineering and Technology, India \\ Rakesh Kasumolla, VNR Vignana Jyothi Institute of Engineering and Technology, India \\ Deepak Sukheja, VNR Vignana Jyothi Institute of Engineering and Technology, India
}

\begin{abstract}
In the last one decade, AI/ML/DL has been considered a core research area in healthcare. As we know that the kidneys are important internal body organs that help in regulation of the fluid within the body such that they relieve the body from the existence of waste. Disease is difficult to detect early on by normal clinical process. Many researchers have focused their work to identify kidney disease or classify kidney disease using computational technology because the mortality rate is very high in kidney patients. The primary focus of this paper is to review the current research work based on computational advancement in the area of kidney disease and also identify the gaps or future scope to improve the process of classifying kidney disease at earlier stage.
\end{abstract}

\section{KEYWORDS}

CADs, Convolutional Neural Network (CNN), DL/AI/ML in Healthcare, Kidney Disease, Medical Image Processing

\section{INTRODUCTION OF CKD}

Initial intervention generally reduces severe disease progress. A report published by Elsevier in February 2020 it shown in figure 1, is indicating the worldwide Growth of Kidney Disease problem is alarming. Near about 8 million peoples are affected by various kinds of kidney related diseases most of kidney disease categorized as Chronic Kidney Disease (CKD) or Acute Kidney Injury (AKI). Chronic Kidney Disease are progressive and non-recoverable in most of cases and Acute kidney injury are $100 \%$ recoverable. But it is also observed that during the treatment of Acute kidney injury around $30 \%$ to $40 \%$ patients also started to suffer the Chronic Kidney Disease (CKD), which is very serious and danger situation for the patients.

Prevalence rates for chronic kidney disease in select countries worldwide in 2017 (per 100,000 population)

CKD is a condition where the gradual loss is being observed in the functionality of the kidneys disallows the purification of the blood. CKD is one of the clinical problems considering the process of dealing with the disease at its final phase and from top to toe likelihood of expiry (Bikbov \& Vos, 2020; Bulletin of the World Health Organization, 2018). Due to kidney disease, according to the WHO (World Health Organization) incidents and deaths reported in 2018 in United States only shown in Figures 2 and 3, respectively. 


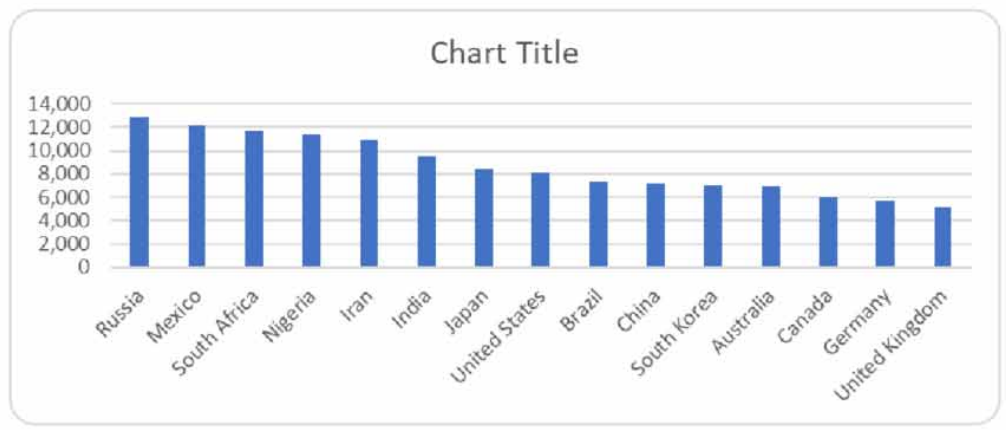

Chronic kidney disease prevalence rates for select countries worldwide 2017 Prevalence rates for chronic kidney disease in select countries worldwide in 2017 (per 100,000 population)

According to a survey by (National Kidney Foundation, 2002), 60\% cases are addressed in undeveloped and developing countries. In Bangladesh around $18 \%$ of the peoples are affected with kidney disease and among them most of the patients are affected with DM (Diabetes Mellitus Type 1) (39.02\%) and DM-2 (Diabetes Mellitus Type 2) (41.46\%). In India, the rise in mortality rate due to chronic diseases has been observed as 3.78 million in 1990 ( $40.4 \%$ of total deaths) to 7.63 million in 2020 .

\subsection{Risk Classification of CKD}

It has been observed that Antivirals, Hyperventilation or Medications and habdomyolysis can also led to kidney injury. Therefore, as per WHO reports many Acute kidney injury cases has been reported during the treatment of COVID-19 also.

Figure 2. Incidents reported in United States from 2010 to 2017

Incidents reported in United States from 2010 to 2017

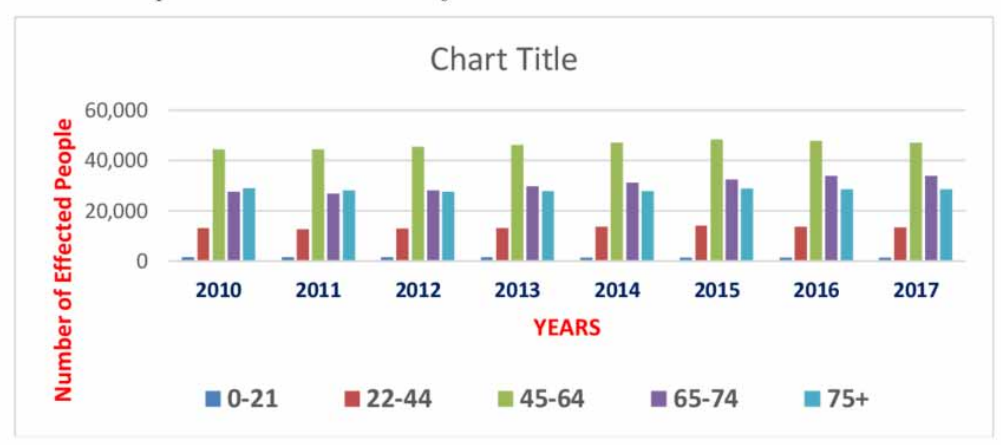


Several articles have been published (Assmann, Cullen, \& Schulte, 2002; Initiative KDOQ. K/doqi clinical practice guidelines on hyper-tension andantihypertensive agents in chronic kidney disease, 2004; Zandi-Nejad, Luyckx, \& Brenner, 2006; Hippisley-Cox et al., 2008; Deng et al., 2017) about CKD risks \& its analysis and authors have observed in their research, hypertension and diabetes are the most common cause of the Chronic Kidney Disease. Apart of hypertension and diabetes, numbers of factors increase the risk of affecting kidneys such as obesity, smoking, old age, high blood pressure (hypertension), certain inherited diseases, family history of cancer and so on. In medical domain, to identify the kidney disease, nephrologist (doctors) suggests the different medical reports based on patient's situation. These reports are urine(urinalysis) key parameters are (RBCs - $£ 2$ RBCs/hpf, WBCs - £2-5 WBCs/hpf), blood tests, imaging tests such as CT scans or MRIs, comprehensive metabolic panel, urine culture, complete blood count, liver or renal panel, Antibiotic Susceptibility Testing and kidney biopsy. Risk Classification of CKD and Evaluation Plans are mentioned in Table 1.

As we know initial intervention generally reduces severe disease progression. In the view of the technological advancements for diagnosing the KD (Kidney Disease) several automated tools can help to identify and classify Kidney Disease Because of in healthcare various tools has been implemented to classify the multiple diseases using AI, Deep Learning and Machine Learning i.e advanced computational technologies can play an important role to predict Kidney Disease at earlier stages.

\section{COMPUTER-AIDED DIAGNOSIS TOOLS AND TECHNIQUE}

Upon reviewing, it is observed that Machine learning, the subset of artificial intelligence could develop relationships using the data where defining them priorly would not be necessary (Deng et al., 2017; Bhaskar \& Manikandan, 2019). Machine learning is classified into three types namely Supervised Learning, whose objective is to learn a mapping from inputs $\mathrm{x}$ to outputs $\mathrm{y}$, input-output pairs $D=\{(X i, Y i)\}_{i=1}^{n}$. Here $\mathrm{D}$ denotes a training set, and $\mathrm{N}$ training examples. Unsupervised Learning is another type in which only inputs are provided $D=\{(X i)\}_{i=1}^{n}$ and to find "interesting patterns" from the data. Reinforcement learning is another type that plays a very crucial role in predicting the behavior of the data.

Deep learning (DL), one of the applications of artificial intelligence, relies on algorithms to process the data and recreation for emerging concepts. It allows computational models that can be composed of multiple processing layers using neural networks that include variety of techniques. The most recognized algorithm, convolutional neural network (CNN) is a part and parcel of Deep Learning models that has become an indispensable process in tasks comprising image detection. In (Khalifa, Taha, Ezzat Ali, Slowik, \& Hassanien, 2020), It states that there are three key methods that successfully work CNNs for image classiðcation: a) training the "CNN from scratch" b) using "offthe-shelf CNN" features extraction as corresponding information channels disease identiðcation and 3) performing ðne-tuning of target images. Architecture for the classification of images is as shown in Figure- 3. Working procedure and functionality of CNN are also well defined in (Khalifa, Taha, Ezzat Ali, Slowik, \& Hassanien, 2020).

CNNs consist of convolutional layers which are characterized by an input map I, a bank of filters $\mathrm{K}$ and biases $\mathrm{b}$. As mentioned by jekfine in their article, If input as type of image with height $\mathrm{H}$, width $\mathrm{W}$ and $\mathrm{C}$ (red, blue, and green) such that $\hat{I I R}^{\mathrm{H} \times \mathrm{W} \times \mathrm{C}}$. Subsequently for a bank of $\mathrm{D}$ filters we have $\mathrm{KIT}^{\mathrm{k} 1 \times \mathrm{k} 2 \times \mathrm{C} \times \mathrm{D}}$ and biases bîR ${ }^{\mathrm{D}}$, one for each filter. Neural Network is a super set of all types of deep learning approaches. The major benefits of neural networks over conventional programming are problems solving ability. Therefore, Neural network technology is seen as cutting-edge today. Neural networks efficiently handle problems like Prediction and pattern recognition. But neural network also has some limitations that it cannot apply neural network or deep learning technique everywhere because of huge complication. To get the higher accuracy of result from deep learning approaches, the size of data set should be very large i.e deep learning approaches are not suggested for small or 
Table 1. Risk classification of CDK and evaluation Plans

\begin{tabular}{|l|l|l|l|}
\hline S. No & \multicolumn{1}{|c|}{ Stages of CIO } & \multicolumn{1}{|c|}{$\begin{array}{c}\text { Glomerular } \\
\text { filtration rate }\end{array}$} & \multicolumn{1}{c|}{ Action Plans } \\
\hline 1 & $\begin{array}{l}\text { Kidney injury with } \\
\text { normal GFR }\end{array}$ & 90 and above & $\begin{array}{l}\text { Analysis of comorbid situations, phylogenies, decrease } \\
\text { in risk }\end{array}$ \\
\hline 2 & $\begin{array}{l}\text { Failure of Kidney with } \\
\text { mild decrease }\end{array}$ & 60 to 89 & Estimate disease evolution \\
\hline 3 & Moderate reduction & 30 to 59 & The Valuation and dealing of ailment problems \\
\hline 4 & Severe lessening & 15 to 29 & $\begin{array}{l}\text { Research in additional treatments like dialysis and } \\
\text { replacement of the kidney for Renal failures. }\end{array}$ \\
\hline 5 & Kidney fiascos & Less than 15 & Kidney standby therapy \\
\hline
\end{tabular}

Figure 3. Death case reported in United Ststes from 2010 to 2017

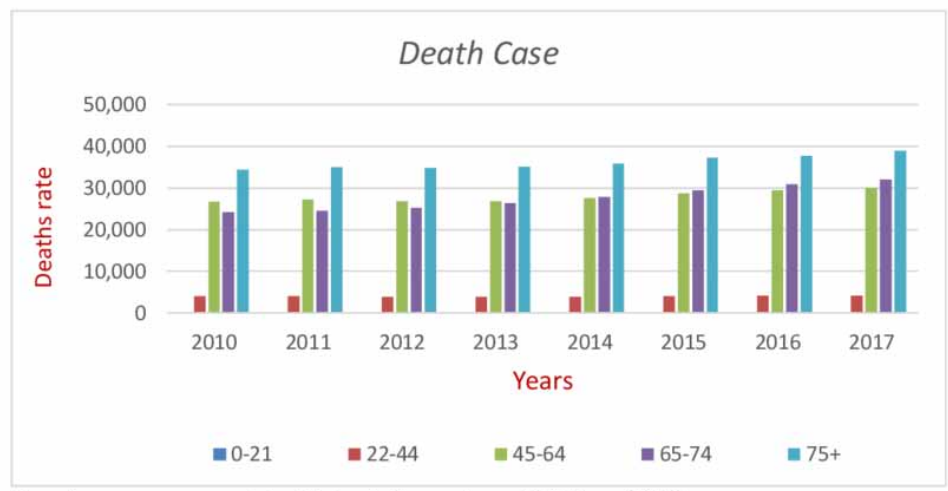

Death case reported in United Ststes from 2010 to 2017

average size datasets owing to the higher levels of training requirements for neural networks. After the major success in 2013, KAIST University in South Korea, Deep Learning experts trained their deep learning model with chest $\mathrm{x}$-rays and mammography images for the detection of lung and breast cancer with an accuracy of $97 \%$. Several researchers have initiated their research in the direction of predicting the kidney disease at earlier stages using AI, Deep Learning, Machine Learning and fuzzy computation.

\section{COMPUTATIONAL TECHNOLOGY GROWTH TOWARDS CKD DIAGNOSIS}

Taking advantage of the information from various types of data for the betterment of the process of diagnosing Deng, Su-Ping, et al. in Deng et al., 2017 mentions the utilization of clinical and pathological features evaluation of Genomic alterations, DNA methylation proðles, RNA and proteomic signatures in KIRC. Gene expression proðles, DNA methylation expression and clinical data are downloaded from TCGA data portal. To predict the cancer stage primarily, network constructed from gene expression data, network constructed from DNA methylation data, secondarily, order to investigate the potential of using networks as a diagnostic tool fused networks (network constructed 


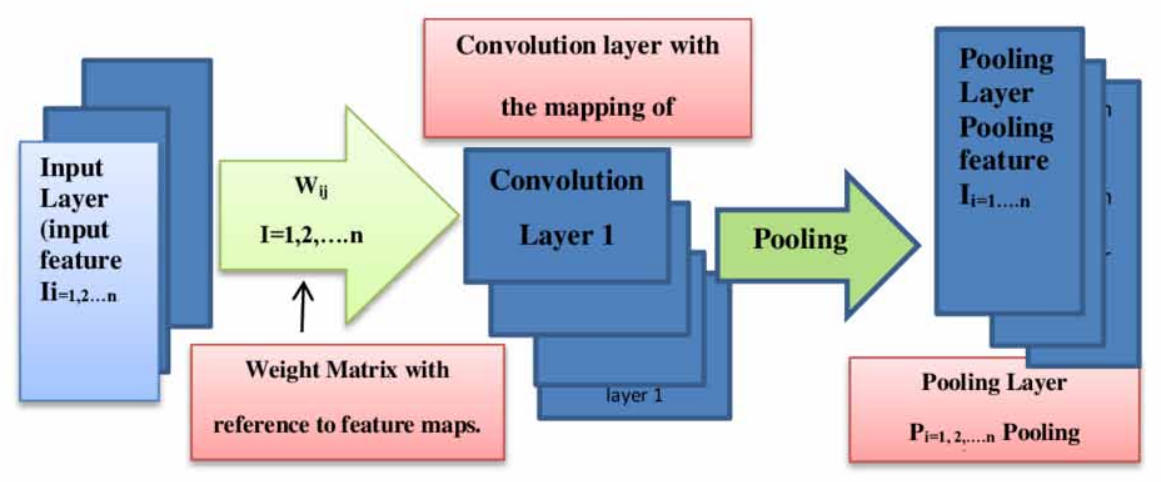

from DNA and network constructed from gene expression) from two data types: gene expression and DNA methylation. In this paper it is claimed that they have achieved a good accuracy in the prediction of the KIRC cancer stage via the fused network. The data taken by the authors is not sufficient, so the authors has used the simulated data which cannot be consider during the experiment/ evaluation of critical disease.

In (Zhang et al., 2019) Hui Zhang, et al., it is suggested that morphological cascade CNNs are based on multi-intersection over union (IOU) threshold in their works. For the identification of minor injuries (1-5 mm), 2 convolution layers in morphology, adapted Feature Pyramid Networks (FPNs) in faster RCNN and combined 4 IOU threshold cascade RCNNs are being implemented. For experimentation, medical CT scan image data set, published by NIH (National Institutes of Health) has been utilized. Experimentation has a requirement of Hardware with Intel Core i5, 2.7 GHz CPU, 8GB RAM that supports Ubuntu, a NVIDIA GTX 1080 video processing card and software's that includes Cascade RCNN deployed in pytorch 1.0 framework, and Faster RCNN used for tensorñow - GPU 1.8. And both python3.5, cuda 9.0 and cudnn 7.1.4. MATLAB based frameworks are utilized. In the work the authors have mentioned about the damage done to the kidney through CNN. As we know that CNN or DL requires large input set but, in this experiment only 985 kidney images were used. which is not enough.

For the prediction of CKD Himanshu Kriplani et al., (Kriplani, Patel, \& Roy, 2019) proposed Deep Neural Network. In 2015 UCI Machine Learning Repository named Chronic Kidney Disease data set is used for proposed Deep Neural Network. The data set comprises of 400 instances, 25 attributes in which 11 are numeric and 14 are nominal. All instances were classified into two categories; 105 has label CKD and 119 has label NCKD (Non CKD). Categories data set is split into two parts 60\% and $40 \%, 60 \%$ used for training purpose and $40 \%$ for testing. With the help of the confusion matrix, the overall performance of the model with an accuracy of $97 \%$ has been measured. The authors mentioned that 18 parameters used during the creation of model but they did not mentioned the details of any parameters.

Navaneeth Bhaskar and Suchetha M et al., in (Bhaskar \& Manikandan, 2019) proposed a new sensing technique for the automatic detection of CKD. The technique is based on a one-dimensional CNN algorithm and SVM classiðer. To test the technique, authors collected 102 samples, including 40 healthy volunteers and 62 individuals with CKD. Samples are dropped via input opening of the chamber for the process of testing which relies on the amount of ammonia gas produced, changes in 
the electrical conductivity of the gas sensor. Deep learning CNN-SVM algorithm is used for automatic computation and classification of the features from the output signal of the sensor.

In (Khalifa, Taha, Ezzat Ali, Slowik, \& Hassanien, 2020), Nour Eldeen M. Khalifa et al suggested a novel optimized approach for the process of classification of 5 various categories of renal cancer, clear cell carcinoma (KIRC), breast invasive carcinoma (BRCA), lung squamous cell carcinoma (LUSC), lung adenocarcinoma (LUAD) and uterine corpus endometrial carcinoma (UCEC) with respect to available images in the dataset. The approach is implemented in 3 phases, preprocessing, augmentation, and deep CNN architecture. Under the preprocessing technique, the high dimensional RNA sequence is optimized to select the optimum number of features using BPSO-DT and then, converts the RNA-Seq into 2D images. The augmentation phase increases the original dataset of 2086 samples to 5 times larger, and finally developed deep CNN architecture to classify KIRC, BRCA, LUSC, LUAD and UCEC cancer. To perform the experiment, researchers used a software package (MATLAB), Intel Xeon E5-2620 processor (2 GHz), 32 GB of RAM and 12 GB Nvidia GTX Titan $\mathrm{X}$ GPU speciðc. To check the accuracy of proposed approach, confusion matrix model is used. Researchers claim minimum $95 \%$ accuracy to classify all the 5 types of cancer.

Ya feng Ren, HaoFe et al presents a hybrid neural network model in (Ren, Fei, Liang, Ji, \& Cheng, 2019). In their works, researchers analyzed the relationship between hypertension and kidney disease. They Collected dataset of patients suffering from diseases or only hypertension. Center points of proposed hybrid neural network model are reimplementation of baseline systems, design the discrete model using Naive Bayes (NB), Support Vector Machine (SVM) and Gradient Boosting Decision Tree (GBDT) and neural network using CNN. The accuracy varies from 64.9 to $81.2 \%$ with respect to different models and features. The Model used in the experiments are LR, NB, SVM and GBDT and feature with respect to every model are Numerical, Textual and Textual-Numerical.

In (Ravizza et al., 2019), it is said that comparison of predictive analytic algorithms using real data could achieve equivalent or enhanced accuracy which is distinguishable with clinical trial data. The accuracy provided for the identification of CKD risk Red, Roche/IBM algorithm is compared with alternative algorithms proposed by Dunkler et al. (Han, Hwang, \& Lee, 2019), Vergouwe et al.(Thong, Kadoury, piche, \& pal, 2018), d Keane et al.(Ravishankar, Prabhu, Vaidya, \& Singhal, 2016) and Jardine et al.(Ravishankar et al., 2016). Roche/IBM algorithm performs better when compared to others. Also, in (Weng, Reps, Kai, Garibaldi, \& Qureshi, 2017) Stephen F. Weng et. Al. tried to assess machine-learning algorithms and check cardiovascular risk prediction which can be improved through ML. complexity, Predictive accuracy and sensitivity of Random forest, logistic regression, gradient boosting machines, neural networks algorithms were compared for the same medical data (24,970 incident cardio-vascular events). In 2017 Geert Litjens et. Al in (Litjens et al., 2017) published an article "A survey on deep learning in medical image analysis" reviewed the major deep learning concepts pertinent to medical image analysis.

Comparison of various research techniques along with their data source and data size and critics shown in table 2.

\section{FINDINGS AND FUTURE DIRECTIONS}

It is believing that artificial intelligent (AI) playing a significant role in the healthcare offerings. Various deep learning and machine learning models based on Computer-Aided Diagnosis techniques for CKD have been reported. Most of the proposed works are based on segmentation via CNN or ML, their effectiveness in terms of the datasets used, although the developed models are learning on smaller datasets. Well-defined large CKD patients (real-time) datasets are still needed. Comparing accuracy of previously reported model by many researchers is highly challenging, due to variances in data set and their characteristics as no direct quantitative metrics or mechanism are available for comparison. Building such common datasets would be a time consuming and a cost ineffective activity. Data and the truth tables would be considered as most significant elements for the consideration to 
Table 2. 4 Year Survey on Technological Growth Toward Ckd Diagnosis

\begin{tabular}{|c|c|c|c|}
\hline $\begin{array}{c}\text { Author Name } \\
\text { and Publication } \\
\text { Year }\end{array}$ & $\begin{array}{l}\text { Applied } \\
\text { Computational } \\
\text { Techniques/ } \\
\text { algorithms }\end{array}$ & $\begin{array}{l}\text { Source of } \\
\text { Dataset }\end{array}$ & Research approach \\
\hline $\begin{array}{l}\text { Ozrgun Cicek et } \\
\text { al.(Olaf, 2016), } \\
2016\end{array}$ & $\begin{array}{l}\text { Neural Network (deepl } \\
\text { learning) }\end{array}$ & $\begin{array}{l}\text { South African } \\
\text { toad Xenopus } \\
\text { laevis }\end{array}$ & $\begin{array}{l}\text { Authors introduced concept of an end-to-end learning, that semi-automatically } \\
\text { and fully-automatically segments a 3D volume from a sparse annotation. It offers } \\
\text { an accurate segmentation for the highly variable structures of the Xenopus kidney. } \\
\text { In this article proposed u-net architecture as an extension for semi- automatic and } \\
\text { full automatic segmented architecture. Accuracy measured in terms of Intersection } \\
\text { over Union (IoU) of their architecture. Researchers collect } 248 \text { sample of "Xenopus } \\
\text { kidney" dataset from South African toad Xenopus laevis in the form of } 132 \times 132 \times \\
116 \text { voxel images and used as an input to proposed architecture with } 3 \text { channels and } \\
\text { output generated voxels images } 44 \times 44 \times 28 \text { at final layer is in x, y, and z directions } \\
\text { Respectively. Weighted softmax loss function is used in training phase and } \\
\text { researcher claimed accuracy is } 0.863 \text { in } 3 \text {-fold cross validation. }\end{array}$ \\
\hline $\begin{array}{l}\text { Xiaoguang Lu et } \\
\text { al.(Lu,Xu, \& Liu,), } \\
2016\end{array}$ & $\begin{array}{l}\mathrm{CNN} \text { and FCN ((deep } \\
\text { learning) }\end{array}$ & Sample Collection & $\begin{array}{l}\text { Research presents a new framework based on CNN and FCN called dual learning } \\
\text { architectures and fusion for organ detection. To localize the organ probabilistic } \\
\text { graphical model in proposed architecture. A right kidney CT body scan image } \\
\text { contains a stack of axial slices used as Inputs in CNN model. } 450 \text { samples were } \\
\text { collected from } 450 \text { patients to train the CNN models. }\end{array}$ \\
\hline Fang Lu et al., 2016 & $\begin{array}{l}\text { Neural Network (deep } \\
\text { learning) and Graph cut }\end{array}$ & $\begin{array}{l}\text { MICCAI-Sliver07 } \\
\text { and } \\
\text { 3Dircadb1 }\end{array}$ & $\begin{array}{l}\text { In this article, 3D deep CNN-based technique is used to identify the liver location } \\
\text { and perform the segmentation task through 3D graph cut based segmentation } \\
\text { refinement. Researcher used } 40 \mathrm{CT} \text { scan images from public data set source available } \\
\text { on MICCAI - Sliver07 and 3Dircadb1. Parameters used in the result analysis are } \\
\text { VOE, RVD, ASD, RMSD and MSD. The proposed technique performed much faster } \\
\text { when compared to manual segmentation system but in number of cases they fail to } \\
\text { segment CT images. Similar kind of task (localization of Anatomical Structures in } \\
\text { 3D Medical Images) is performed on different dataset with a different approach. }\end{array}$ \\
\hline $\begin{array}{l}\text { Hariharan } \\
\text { Ravishankar et al., } \\
2016\end{array}$ & $\begin{array}{l}\text { Neural Network (deep } \\
\text { learning) }\end{array}$ & $\begin{array}{l}\text { LOGIQ E9 } \\
\text { scanner }\end{array}$ & $\begin{array}{l}\text { This article mentions the implementation of a new hybrid deep CNN model with } \\
\text { CaffetNet features to automatically segment and quantity abdominal shape in foetal } \\
\text { ultrasound images. In this work, authors used Ultrasound data as an input for their } \\
\text { model. Sample size used is } 90 \text { and source of input (Ultrasound data) was LOGIQ } \\
\text { E9 scanner https://www.gehealthcare.in/products/ultrasound/logiq/logiq-e9-with- } \\
\text { xdclear). Merits of deep learning } \\
\text { and conventional features are also explored in this article. }\end{array}$ \\
\hline $\begin{array}{l}\text { Hariharan } \\
\text { Ravishankar et al., } \\
2017\end{array}$ & $\begin{array}{l}\text { Neural Network (deep } \\
\text { learning) }\end{array}$ & $\begin{array}{l}\text { 2-D fetal } \\
\text { ultrasound images }\end{array}$ & $\begin{array}{l}\text { This article presents the extended work and proposed a hybrid approach combining } \\
\text { traditional texture analysis methods using deep learning and HOG - GBM features. } \\
70 \text { samples of 2-D foetal ultrasound images used to check accuracy of proposed } \\
\text { technique. To avoid the vanishing gradient problem, they used ReLU as the activation } \\
\text { function. }\end{array}$ \\
\hline $\begin{array}{l}\text { William Thong } \\
\text { et al., } \\
2016\end{array}$ & $\begin{array}{l}\text { Neural Network (deepl } \\
\text { learning) }\end{array}$ & Sample Collection & $\begin{array}{l}\text { To diagnosis kidney disease, fully automatic framework presents for kidney } \\
\text { segmentation using CNN. To predict the class membership model trained through } \\
\text { patch-wise approach. } 3 \text { random subsets were generated from } 79 \text { sample data. Present } \\
\text { framework is fully based on } 2 \text { D inputs and evaluation of segmentation is based on } \\
\text { linear, Nearest Neighbour, Bilinear interpolation methods. }\end{array}$ \\
\hline $\begin{array}{l}\text { Su-Ping Deng et al., } \\
2017\end{array}$ & $\begin{array}{l}\text { Network fusion method } \\
\text { and Generalized linear } \\
\text { model. }\end{array}$ & TCGA & $\begin{array}{l}\text { Proposed a novel concept called Network fusion method. The work done is about } \\
\text { the cancer stage prediction in the kidney. To predict the label of a new sample semi- } \\
\text { supervised learning is used. The results have proved that Network-based LASSO } \\
\text { Label Prediction (NLLP) method has good potential. Major problem with this work } \\
\text { is results have generated through simulated data. Simulated data cannot be trusted for } \\
\text { treatment in a dangerous disease like cancer. }\end{array}$ \\
\hline $\begin{array}{l}\text { Stephen F. Weng } \\
\text { et al., } \\
2017\end{array}$ & $\begin{array}{l}\text { Machine-learning } \\
\text { Algorithms }\end{array}$ & $\begin{array}{l}\text { Clinical Practice } \\
\text { Research Data } \\
\text { link (CPRD) }\end{array}$ & $\begin{array}{l}\text { Compared different established machine-learning algorithms like random forest, } \\
\text { logistic regression, gradient boosting machines, and neural networks to predict first } \\
\text { cardiovascular event over } 10 \text {-years. Comparison was performed on } 3,78,256 \mathrm{CPRD} \\
\text { dataset and findings risk prediction improved by random forest }+1.7 \% \text {, logistic } \\
\text { regression }+3.2 \% \text {, neural networks }+3.6 \% \text { and by gradient boosting }+3.3 \% \text {. This risk } \\
\text { prediction is based on } 29 \text { parameters. }\end{array}$ \\
\hline $\begin{array}{l}\text { Timothy L. Kline } \\
\text { et al., } \\
2017\end{array}$ & Deep Neural Network & TEMPO study & $\begin{array}{l}\text { Described a fully automated deep Neural Network approach for Fully Automated } \\
\text { Segmentation of Polycystic Kidneys disease. Researchers simulated MR Image with } \\
\text { multi-observer approach to predict an accurate result. } 2000 \text { DICOM image dataset } \\
\text { is used in Deep Neural Network. Proposed approach offered effective process to } \\
\text { measure the TKV imaging biomarker for kidneys patients. }\end{array}$ \\
\hline
\end{tabular}


Table 2. Continued

\begin{tabular}{|c|c|c|c|}
\hline $\begin{array}{l}\text { Author Name } \\
\text { and Publication } \\
\text { Year }\end{array}$ & $\begin{array}{l}\text { Applied } \\
\text { Computational } \\
\text { Techniques/ } \\
\text { algorithms }\end{array}$ & $\begin{array}{c}\text { Source of } \\
\text { Dataset }\end{array}$ & Research approach \\
\hline $\begin{array}{l}\text { Eli Gibson et al., } \\
2018\end{array}$ & Deep Learning & TCIA and BTCV & $\begin{array}{l}\text { To segment multiple organs, proposed a registration-free deep-learning-based } \\
\text { segmentation algorithm based on dilated convolutions, dense feature stacks, } \\
\text { batch-wise spatial dropout, up-sampling and V-network down-sampling. During } \\
\text { the experimental study, liver, gallbladder, spleen and left kidney types of organs } \\
\text { have been segmented. Adam optimizer is used to train the network. In this study, } \\
\text { ninety abdominal CT images of the spleen, left kidney, gallbladder, esophagus, liver, } \\
\text { stomach, pancreas and duodenum are used. Proposed technique is compared with } \\
\text { deep-learning-based VoxResNet and MALF-based DEEDS +JLF pro. }\end{array}$ \\
\hline $\begin{array}{l}\text { Hui Zhang et al., } \\
2019\end{array}$ & $\begin{array}{l}\text { Morphological image } \\
\text { processing technique } \\
\text { and Neural Network } \\
\text { (deep learning) }\end{array}$ & $\begin{array}{l}\text { Deep Lesion } \\
\text { (PACSs) }\end{array}$ & $\begin{array}{l}\text { Author proposed a Framework of kidney lesion detection, that mainly includes two } \\
\text { steps: first acquisition of six different sizes lesions detection feature maps and second } \\
\text { multi-IOU cascade RCNN. In the work the authors have mentioned about the damage } \\
\text { done to the kidney through CNN. As we know that CNN or DL requires large input } \\
\text { set but, in this experiment only } 985 \text { kidney images were used. which is not enough }\end{array}$ \\
\hline $\begin{array}{l}\text { Navaneeth Bhaskar } \\
\text { et al., } \\
2019\end{array}$ & $\begin{array}{l}\text { Machine learning } \\
\text { technique and Neural } \\
\text { Network (deep learning) }\end{array}$ & Sample Collection & $\begin{array}{l}\text { Proposed new electronics-based sensing mechanism for the automated detection } \\
\text { of kidney disease, and it monitors the urea levels in the saliva sample. The sensing } \\
\text { mechanism consists of a gas sensing chamber, Arduino board and an MQ-series } \\
\text { ammonia gas sensor. The conversion reaction is carried out inside the gas chamber. } \\
\text { Testing is performed by dropping the sample through the input opening of the } \\
\text { chamber for normal sample sensor produces a voltage of } 0.62-0.93 \mathrm{~V} \text {. Their results } \\
\text { are promising with good accuracy but there is need for sample testing towards } \\
\text { accuracy of analysis of this approach. }\end{array}$ \\
\hline $\begin{array}{l}\text { Yafeng Ren et al., } \\
2019\end{array}$ & Hybrid neural network & $\begin{array}{l}\text { EHR Data of } 12 \\
\text { Hospitals (2012- } \\
\text { 2017) }\end{array}$ & $\begin{array}{l}\text { Proposed Hybrid neural model based on (BiLSTM) and Auto encoder networks } \\
\text { for identifying kidney disease in hypertension patients. In proposed model mainly } \\
\text { includes BiLSTM and Autoencoder. BiLSTM is used for learning and deal with } \\
\text { textual description information and Autoencoder deal with physical indicators. Used } \\
35,332 \text { HER data sample to check the accuracy of implemented model. The proposed } \\
\text { model may be used for coronary heart disease prediction in hypertension patients. }\end{array}$ \\
\hline $\begin{array}{l}\text { Stefan Ravizza } \\
\text { et al., } \\
2019\end{array}$ & Roche/IBM algorithm & $\begin{array}{l}\text { IBM Explorys } \\
\text { database }\end{array}$ & $\begin{array}{l}\text { Researchers presents a novel Roche/IBM algorithm, implemented in IBM lab. } \\
\text { Algorithm was tested on 550,00,000 INPC huge data set. Roche/IBM algorithm is } \\
\text { used over the real-world data and achieved the good accuracy }\end{array}$ \\
\hline $\begin{array}{l}\text { Seokmin Han et al., } \\
2019\end{array}$ & Deep Learning & $\begin{array}{l}\text { Seoul National } \\
\text { University } \\
\text { Bundang Hospital }\end{array}$ & $\begin{array}{l}\text { Proposed deep learning approach using convolutional neural network and ROI for } \\
\text { three-layer classification to classify the renal cancer in kidney. In the article, crucial } \\
\text { information about data set is missing. }\end{array}$ \\
\hline $\begin{array}{l}\text { Nour Eldeen M. } \\
\text { Khalifa et al., } \\
2020\end{array}$ & Deep learning approach & $\begin{array}{l}\text { Tumor gene } \\
\text { expression dataset }\end{array}$ & $\begin{array}{l}\text { Authors have proposed a common framework in his research work to classify five } \\
\text { different types of cancer. To know the accuracy and precision of the proposed } \\
\text { framework, the authors have used } 2086 \text { sample, in which KIRC (kidney related } \\
\text { dataset) number is only 537, it is not enough for a deep learning techniques. }\end{array}$ \\
\hline $\begin{array}{l}\text { Shi Yin et al., } \\
2020\end{array}$ & $\begin{array}{l}\text { Boundary distance } \\
\text { regression andPixel } \\
\text { classification networks }\end{array}$ & US images & $\begin{array}{l}\text { Proposed boundary distance regression and Pixel classification networks approach. } \\
\text { The approach is good but has average performance and significantly better than deep } \\
\text { learning-based pixel classification networks. To check the performance } 185 \text { sample } \\
\text { of Kidney Pole images were used. }\end{array}$ \\
\hline $\begin{array}{l}\text { Ho Sun Shon et al., } \\
2020\end{array}$ & Deep Learning & TCGA & $\begin{array}{l}\text { Proposed an end-to-end, cost-sensitive hybrid deep learning (COST-HDL) approach } \\
\text { with a cost-sensitive loss functions. This approach is more efficient compared to } \\
\text { traditional data mining techniques and conventional machine learning. To check the } \\
\text { efficiency authors used Gene expression data with } 1157 \text { sample size. }\end{array}$ \\
\hline Guozhen Chen et al. & $\begin{array}{l}\text { Neural Network (deep } \\
\text { learning) }\end{array}$ & $\begin{array}{l}\text { miRNA genome } \\
\text { data }\end{array}$ & $\begin{array}{l}\text { Authors has proposed Adaptive hybridized Deep Convolutional Neural Network } \\
\text { (CNN) for the early detection of Kidney disease. authors have used datasets that } \\
\text { present at https://www.mediafire.com/. The proposed AHDCNN explore the } \\
\text { consistent renal cell rating forecasts from CT (CECT) images. ACCURACY } \\
\text { ANALYSIS is well defined in result experiments. Efðciency of the proposed model } \\
\text { at all classiðcation thresholds level is explained through ROC curve. }\end{array}$ \\
\hline
\end{tabular}


apply deep learning or machine learning methods. The inadequate datasets are a major problem to further advancement of Computer-Aided Diagnosis techniques and models In Chronic Kidney Disease (CKD). Finally, upon reviewing the various literature, it can be said that the prediction of CKD can be improved in different ways only with the development of machine learning or deep learning models. It can also be made possible through exploring the performance-complementary properties (Hybrid Model) using a combination of deep learning model, fuzzy logic and statistics techniques (lot of classical statistical methodology are present through which establish an equation or curve) for scratch medical image, pathological microscopic image and text data dataset.

\section{CONCLUSION}

Computer-Aided Diagnosis Tools and Technique for Chronic Kidney Disease have adept various domains including medical research with magnificent achievements, and an interest has emerged progressively in radiology. Although most of researcher have focused on deep learning method to classify the CKD and proposed the good model also. Most of the research is facilitating learning on smaller datasets because of the non-availability of sufficient dataset. one more interesting factor is maximum research is based on medical image data, no one have considered the pathological dataset during their research. As per doctor opinion to predict the kidney diseases at right time or earlier stage pathological report behavior of patient must be observed. Sometime "pathological based data" indicate abnormality at earlier stage but to correctly classify the abnormality medical images have been required. Similarly, sometime medical images indicate abnormality at earlier stage but to correctly classify the abnormality pathological based data have been required. As a research point of view, lot of scope is available to identify and classify kidney disease using advanced computational techniques.

Although deep learning has become a dominant method to classify the CKD and several highproðle successes of deep learning and machine learning technique have been reported in table 2, most of the research is facilitating learning on smaller datasets. 


\section{REFERENCES}

Alonso, A., Lau, J., Jaber, B. L., Weintraub, A., \& Sarnak, M. J. (2004). Prevention of radiocontrast nephropathy with $\mathrm{N}$-acetylcysteine in patients with chronic kidney disease: A meta-analysis of randomized, controlled trials. American Journal of Kidney Diseases, 43(1), 1-9. doi:10.1053/j.ajkd.2003.09.009 PMID:14712421

Assmann, G., Cullen, P., \& Schulte, H. (2002). Simple scoring scheme for calculating the risk of acute coronary events based on the 10-year fol-low-up of the prospective cardiovascular mnster (procam) study. Circulation, 105(3), 310-315. doi:10.1161/hc0302.102575 PMID:11804985

Bhaskar, N., \& Manikandan, S. (2019). A deep-learning-based sys-tem for automated sensing of chronic kidney disease. IEEE Sensors Letters. Letters, 3(10), 1-4. 10.1109/LSENS.2019.2942145

Bikbov, B., \& Vos, T. (2020). Global, regional, and national burden of chronic kidney disease, 1990-2017: A systematic analysis for the Global Burden of Disease Study. Academic Press.

Chen, G., Ding, C., Li, Y., Hu, X., Li, X., Ren, L., Ding, X., Tian, P., \& Xue, W. (2020). Prediction of chronic kidney disease Using Adaptive Hybridized Deep Convolutional Neural Network on the in-ternet of medical things platform. IEEE Access: Practical Innovations, Open Solutions, 8, 100497-100508. doi:10.1109/ ACCESS.2020.2995310

Chen, H., Dou, Q., Yu, L., Qin, J., \& Heng, P. A. (2018). VoxResNet: Deep voxelwise residual networks for brain segmentation from 3D MR images. NeuroImage, 170, 446-455. doi:10.1016/j.neuroimage.2017.04.041 PMID:28445774

de Vos, B. D., Wolterink, J. M., de Jong, P. A., Leiner, T., Viergever, M. A., \& Išgum, I. (2017). ConvNet-based localization of anatomical structures in 3-D medical images. IEEE Transactions on Medical Imaging, 36(7), 1470-1481. .10.1109/TMI.2017.2673121

Deng, S.-P., Cao, S., Huang, D. S., \& Wang, Y. P. (2017). Identifying stages of kidney renal cell carcinoma by combining gene expression and DNA methylation data. IEEE/ACM Transactions on Computational Biology and Bioinformatics, 14(5), 1147-1153. doi:10.1109/TCBB.2016.2607717 PMID:28113675

Gibson, E., Giganti, F., Hu, Y., Bonmati, E., Bandula, S., Gurusamy, K., Davidson, B., Pereira, S. P., Clarkson, M. J., \& Barratt, D. C. (2018). Automatic multi-organ seg-mentation on abdominal CT with dense V-networks. IEEE Transactions on Medical Imaging, 37(8), 1822-1834. doi:10.1109/TMI.2018.2806309 PMID:29994628

Han, S., Hwang, S. I., \& Lee, H. J. (2019). The classification of renal cancer in 3-phase CT images using a deep learning method. Journal of Digital Imaging, 32(4), 638-643. doi:10.1007/s10278-019-00230-2 PMID:31098732

Hippisley-Cox, J., Coupland, C., Vinogradova, Y., Robson, J., Minhas, R., Sheikh, A., \& Brindle, P. (2008). Predicting cardiovascular risk in England and wales:prospective derivation and validation of qrisk2. BMJ (Clinical Research Ed.), 336(7659), 1475-1482. doi:10.1136/bmj.39609.449676.25 PMID:18573856

Jiang, J., Trundle, P., \& Ren, J. (2010, December). Medical image analysis with artiðcial neural networks. Computerized Medical Imaging and Graphics, 34(8), 617-631. doi:10.1016/j.compmedimag.2010.07.003 PMID:20713305

Kakitapalli, Y., Ampolu, J., Madasu, S. D., \& Sai Kumar, M. L. S. (2020). Detailed review of chronic kidney disease. Kidney Diseases, 6(2), 85-91. doi:10.1159/000504622 PMID:32309290

Khalifa, N. E. M., Taha, M. H. N., Ezzat Ali, D., Slowik, A., \& Hassanien, A. E. (2020). Artificial intelligence technique for gene expression by tumor RNA-seq data: A novel optimized deep learning approach. IEEE Access: Practical Innovations, Open Solutions, 8, 22874-22883. doi:10.1109/ACCESS.2020.2970210

Kline, T. L., Korfiatis, P., Edwards, M. E., Blais, J. D., Czerwiec, F. S., Harris, P. C., King, B. F., Torres, V. E., \& Erickson, B. J. (2017). Performance of an artificial multi-observer Deep Neural Network for Fully Automated Segmentation of Polycystic Kidneys. Journal of Digital Imaging, 30(4), 442-448. doi:10.1007/s10278-0179978-1 PMID:28550374

Kriplani, H., Patel, B., \& Roy, S. (2019). Prediction of chronic kidney diseases using deep artificial neural network technique. .10.1007/978-3-030-04061-1_18 
Litjens, G., Kooi, T., Bejnordi, B. E., Setio, A. A. A., Ciompi, F., Ghafoorian, M., van der Laak, J. A. W. M., van Ginneken, B., \& Sánchez, C. I. (2017). A survey on deep learning in medical image analysis. Medical Image Analysis, 42, 60-88. doi:10.1016/j.media.2017.07.005 PMID:28778026

Lu, F., Wu, F., Hu, P., Peng, Z., \& Kong, D. (2017). Automatic 3D liver location and segmentation via convolutional neural network and graph cut. International Journal of Computer Assisted Radiology and Surgery, 12(2), 171-182. doi:10.1007/s11548-016-1467-3 PMID:27604760

Lu, X., Xu, D., \& Liu, D. (2016). Robust 3D organ localization with dual learning architectures and fusion. Lecture Notes in Computer Science, 10008, 12-20. doi:10.1007/978-3-319-46976-8_2

Luyckx, V. A., Tonelli, M., \& Stanifer, J. W. (2018). The global burden of kidney disease and the sustainable development goals. Bulletin of the World Health Organization, 96(6), 414-422D. 10.2471/BLT.17.206441

Miotto, R., Wang, F., Wang, S., Jiang, X., \& Dudley, J. T. (2018). Deep learning for healthcare: Review, opportunities and challenges [Review]. Briefings in Bioinformatics, 19(6), 1236-1246. doi:10.1093/bib/bbx044 PMID:28481991

Murphy Kevin, P. (2012). Machine learning: A probabilistic perspective. MIT Press.

Noll, M., Li, X., \& Wesarg, S. (2014). Automated kidney detection and segmentation in 3D ultrasound. In Proceedings of the Workshop Clin. Image-Based Procedures (pp. 83-90). Academic Press.

Olaf. (2016). Çiçek, Özgün and Abdulkadir, Ahmed and Lienkamp. In U-Net: Learning Dense Volumetric Segmentation from Sparse Annotation. Academic Press.

Ravishankar, H., Prabhu, S. M., Vaidya, V., \& Singhal, N. (2016). Hybrid approach for automatic segmentation of fetal abdomen from ultrasound images using deep learning. In IEEE 13th International Symposium on Biomedical Imaging (ISBI), Prague, 2016 (pp. 779-782). doi:10.1109/ISBI.2016.7493382

Ravishankar, H., Sudhakar, P., Venkataramani, R., Thiruvenkadam, S., Annangi, P., Babu, N., \& Vaidya, V. (2016). Understanding the mechanisms of deep transfer learning for medical images. Lecture Notes in Computer Science, 188-196. doi:10.1007/978-3-319-46976-8_20

Ravizza, S., Huschto, T., Adamov, A., Böhm, L., Büsser, A., Flöther, F. F., Hinzmann, R., König, H., McAhren, S. M., Robertson, D. H., Schleyer, T., Schneidinger, B., \& Petrich, W. (2019). Predicting the early risk of chronic kidney disease in patients with diabetes using real-world data. Nature Medicine, 25(1), 57-59. doi:10.1038/ s41591-018-0239-8 PMID:30617317

Ren, Y., Fei, H., Liang, X., Ji, D., \& Cheng, M. (2019). A hybrid neural network model for predicting kidney disease in hypertension patients based on electronic health records. BMC Medical Informatics and Decision Making, 19(Suppl 2), 51. 10.1186/s12911-019-0765-4

Shin, H. C., Roth, H. R., Gao, M., Lu, L., Xu, Z., Nogues, I., Yao, J., Mollura, D., \& Summers, R. M. (2016). Deep convolutional neural networks for computer-aided detection: CNN architectures, dataset characteristics and transfer learning. IEEE Transactions on Medical Imaging, 35(5), 1285-1298. doi:10.1109/TMI.2016.2528162 PMID:26886976

Shon, H. S., Batbaatar, E., Kim, K. O., Cha, E. J., \& Kim, K. (2020). Classification of Kidney Cancer Data Using Cost-Sensitive Hybrid Deep Learning Approach. Symmetry, 12(1), 154. doi:10.3390/sym12010154

Simmons, M. N., Ching, C. B., Samplaski, M. K., Park, C. H., \& Gill, I. S. (2010). Kidney tumor location measurement using the c index method. The Journal of Urology, 183(5), 1708-1713. doi:10.1016/j. juro.2010.01.005 PMID:20299047

Thong, W., Kadoury, S., Piché, N., \& Pal, C. J. (2018). Convolutional networks for kidney segmentation in contrast-enhanced CT scans. Computer Methods in Biomechanics and Biomedical Engineering. Imaging \& Visualization, 6(3), 277-282. doi:10.1080/21681163.2016.1148636

Wang, H., Suh, J. W., Das, S. R., Pluta, J. B., Craige, C., \& Yushkevich, P. A. (2013). Multi-atlas segmentation with joint label fusion. IEEE Transactions on Pattern Analysis and Machine Intelligence, 35(3), 611-623. doi:10.1109/TPAMI.2012.143 PMID:22732662 
Weng, S. F., Reps, J., Kai, J., Garibaldi, J. M., \& Qureshi, N. (2017). Can machine-learning improve cardiovascular risk prediction using routine clinical data? PLoS One, 12(4), e0174944. doi:10.1371/journal.pone.0174944 PMID:28376093

Yamashita, R., Nishio, M., Do, R. K. G., \& Togashi, K. (2018). Convolutional neu-ral networks: An overview and application in radiology. Insights Into Imaging, 9(4), 611-629. doi:10.1007/s13244-018-0639-9 PMID:29934920

Yin, S., Peng, Q., Li, H., Zhang, Z., You, X., Fischer, K., Furth, S. L., Tasian, G. E., \& Fan, Y. (2020). Automatic kidney segmentation in ultrasound images using subsequent boundary distance regression and pixelwise classification networks. Medical Image Analysis, 60, 101602. doi:10.1016/j.media.2019.101602 PMID:31760193

Zandi-Nejad, K., Luyckx, V. A., \& Brenner, B. M. (2006). Adult hypertension and kidneydisease the role of fetal programming. Hypertension, 47(3), 502-508. doi:10.1161/01.HYP.0000198544.09909.1a PMID:16415374

Zeng, Z. Q. (2008). Fast training Support Vector Machines using parallel sequential minimal optimization. In Intelligent System and Knowledge Engineering. ISKE 2008. 3rd International Conference on Volume Abteilung 1. IEEE.

Zhang, H., Chen, Y., Song, Y., Xiong, Z., Yang, Y., \& Jonathan Wu, Q. M. J. (2019). Automatic kidney lesion detection for CT images us-ing morphological cascade convolutional neural networks. IEEE Access, 7 , 83001-83011.

Mynapati Lakshmi Prasudha completed M. Tech from VNRVJIET and presently is doing research in the areas of Machine learning and Deep learning. Rakesh Kasumolla is an M. Tech Student at VNR VJIET. Deepak Sukheja is working as an Associate professor in the Department of Computer Science and Engineering at VNRVJIET (NAAC accredited A++ Grade, one among top 5 colleges of Hyderabad), Hyderabad, India. Deepak obtained a Ph.D. in Computer Science from Vikram University, Ujjain in 2014, Master of Technology degree in Computer Technology from Govt. Engineering College (NIT) Raipur (CG)-2005, and Master in Computer Science from (DAVV) Indore in 1999. Deepak has published more than 22 research papers, published two patent and is working as a reviewer for Elsevier Journals and others. Along with academics, Deepak has served his services as a senior software engineer to Patni Computer and Wipro Technology during the period of 2004 to 2007. His current research interests include Data Science, Deep learning, Data Analytics, Blockchain Technology, Query Optimization and Distributed System. 